\title{
The first INTEGRAL-OMC catalogue of optically variable sources ^
}

\author{
J. Alfonso-Garzón ${ }^{1}$, A. Domingo ${ }^{1}$, J. M. Mas-Hesse ${ }^{1}$, and A. Giménez ${ }^{2,1}$ \\ ${ }^{1}$ Centro de Astrobiología, Departamento de Astrofísica (CSIC-INTA), POB 78, 28691 Villanueva de la Cañada, Spain \\ e-mail: julia@cab.inta-csic.es \\ ${ }^{2}$ European Space Astronomy Centre (ESAC), European Space Agency, POB 78, 28691 Villanueva de la Cañada, Spain
}

Received 24 July 2012 / Accepted 28 September 2012

\section{ABSTRACT}

\begin{abstract}
Context. The Optical Monitoring Camera (OMC) onboard INTEGRAL provides photometry in the Johnson $V$-band. With an aperture of $50 \mathrm{~mm}$ and a field of view of $5^{\circ} \times 5^{\circ}$, OMC is able to detect optical sources brighter than $V \sim 18$, from a previously selected list of potential targets of interest. After more than nine years of observations, the OMC database contains light curves for more than 70000 sources (with more than 50 photometric points each).

Aims. The objectives of this work have been to characterize the potential variability of the objects monitored by OMC, to identify periodic sources and to compute their periods, taking advantage of the stability and long monitoring time of the OMC.

Methods. To detect potential variability, we have performed a chi-squared test, finding 5263 variable sources out of an initial sample of 6071 objects with good photometric quality and more than 300 data points each. We have studied the potential periodicity of these sources using a method based on the phase dispersion minimization technique, optimized to handle light curves with very different shapes.

Results. In this first catalogue of variable sources observed by OMC, we provide for each object the median of the visual magnitude, the magnitude at maximum and minimum brightness in the light curve during the window of observations, the period, when found, as well as the complete intrinsic and period-folded light curves, together with some additional ancillary data.
\end{abstract}

Key words. catalogs - astronomical databases: miscellaneous - stars: variables: general - techniques: photometric

\section{Introduction}

The study of variable stars is one of the principal areas of astronomical research. The beginning of their study dates back to the 16 th century when the first variable star, a supernova, was identified, and shortly thereafter telescopes started to be used for astronomy. Since then, an increasing number of variable stars were discovered and observed but it was with the upcoming of first photography and photoelectric photometry and later of the CCD technology and multi-wavelength astronomy that the number of known variable stars increased very rapidly. In 1948 the first edition of the General Catalogue of Variable Stars (GCVS) was published containing 10820 stars. The GCVS has been updated regularly until its last release (Samus et al. 2012). The number of known variable stars has increased up to around $200000 \mathrm{ob}-$ jects compiled in the Variable Star Index (VSX; Watson 2006; Watson et al. 2012). The VSX is maintained by the American Association of Variable Stars Observers (AAVSO), and contains all objects from the GCVS and the catalogues of the Northern Sky Variability Survey (NSVS; Woźniak et al. 2004), as well as data resulting from other automated surveys, like the All Sky Automated Survey (ASAS; Pojmanski 1997), or the catalogues compiled from the Optical Gravitational Lensing Experiment (OGLE; Udalski et al. 1992) observations: the Miras and eclipsing binaries from OGLE-II data or the catalogue of variable stars from OGLE-III.

* List of object data and full set of figures with finder charts and light curves are only available at the CDS via anonymous ftp to cdsarc.u-strasbg.fr $(130.79 .128 .5)$ or via http://cdsarc.u-strasbg.fr/viz-bin/qcat?]/A+A/548/A79
The advent of space astronomy has provided additional high quality data for thousands of objects. The most significant impact has been produced by the missions specifically dedicated to obtain photometric data of high accuracy, in some cases during very long periods of time. While optimized for astrometric studies, the HIPPARCOS/Tycho mission also produced high accuracy photometric time series (Perryman et al. 1997; Hoeg et al. 1997). Even higher photometric accuracy is provided by dedicated missions, aimed to study asteroseismology or to search for exoplanetary transits, like the Canada's Microvariability and Oscillations of STars (MOST; Walker et al. 2003), the Wide-field Infrared Explorer (WIRE; Bruntt \& Buzasi 2006), the COnvection, ROtation and planetary Transits (CoRoT) mission (Auvergne et al. 2009) or Kepler (Koch et al. 2010), which is yielding the highest accuracy light curves available up to now.

Furthermore, spacecraft systems or secondary instruments on the satellites can provide very useful photometric information as well. Zwintz et al. (2000) performed a detailed analysis of more than 4500 data sets from the Hubble Space Telescope (HST) guide star data, finding 20 stars to be variable. In a similar way, the observations performed by the spacecraft tracking cameras of Chandra have allowed to produce the Chandra Variable Guide Star Catalogue (VGUIDE) containing data of 827 stars for which accurate, long term photometry was available (Nichols et al. 2010).

The International Gamma-Ray Astrophysics Laboratory (INTEGRAL) mission, launched October 17th, 2002, Winkler et al. (2003) includes a small optical telescope, the Optical Monitoring Camera (OMC), to provide photometry in the Johnson $V$-band simultaneously to the high-energy observations (Mas-Hesse et al. 2003). In addition to monitoring the primary 
high-energy targets, OMC has been observing serendipitously tens of thousands of potentially variable objects within its field of view $(\mathrm{FoV})$, preselected from existing catalogues of variable stars.

In this paper we present the first INTEGRAL-OMC catalogue of optically variable sources (OMC-VAR hereafter) that contains photometric data in the $V$-band, as well as variability and periodicity data for more than 5000 objects observed from October 2002 to February 2010. The catalogue will be updated as new data are being analysed. At the end of the mission we expect to produce the final catalogue with variability information for more than 25000 objects monitored over a long time period, with consistent and well calibrated photometry from space.

\section{The Optical Monitoring Camera onboard INTEGRAL}

The OMC is a small refractive telescope with an aperture of $50 \mathrm{~mm}$ focused onto a large format CCD $(1024 \times 2048$ pixels) working in frame transfer mode $(1024 \times 1024$ pixels imaging area). With a FoV $5^{\circ} \times 5^{\circ} \mathrm{OMC}$ provides photometry in the Johnson $V$-band (centred at $5500 \AA$ ) and for sources from $V \sim 7 \mathrm{mag}$ (for brighter sources saturation effects appear) to $V \sim 16-17$ mag (limit magnitude for $3 \sigma$ source detection and $200 \mathrm{~s}$ integration time). Typical observations are performed as a sequence of different integration times, allowing for photometric uncertainties below 0.1 mag for objects with $V \leq 16$. The accuracy is limited to $0.01 \mathrm{mag}$ by the flat field correction matrix. OMC was designed to observe the optical emission from the prime targets of the gamma-ray instruments onboard INTEGRAL and has the same FoV as the fully coded FoV of the INTEGRAL X-ray monitor (JEM-X; Lund et al. 2003), and it is co-aligned with the central part of the gamma-ray instruments larger fields of view, the IBIS imager (Ubertini et al. 2003) and the SPI spectrometer (Vedrenne et al. 2003). The need to have a FoV as large as possible led to a rather large angular pixel size $\left(17^{\prime \prime} .5 \times 17^{\prime \prime} .5\right)$, with a Gaussian Point Spread Function with $F W H M \sim 1.4$ pix.

\subsection{The OMC input catalogue}

In addition to monitoring the INTEGRAL primary high-energy targets, OMC has the capability to observe serendipitously around 100 sources within its FoV. These targets have to be preselected on ground, since the limited telemetry available to OMC (only $\sim 2.2 \mathrm{kbps}$ ) does not allow to download full images to ground. These additional targets are automatically selected from the OMC Input Catalogue (OMC-IC hereafter) by a specific OMC Pointing Software (OMCPS) running at the INTEGRAL Science Operation Centre (ISOC). Only $11 \times 11$ pix windows of the CCD containing those objects are transmitted to ground. The OMC-IC was compiled before the start of the mission, and is described in detail in Domingo et al. (2003). It has been updated several times to include new targets of interest or to improve the accuracy in coordinates, especially of the high-energy objects.

The current version of the OMC-IC contains over 500000 entries, namely:

- astrometric and photometric reference stars;

- known optical counterparts of gamma-ray sources;

- known optical counterparts of X-ray sources;

- point-like X-ray sources detected and catalogued by ROSAT;

- quasars observable by the OMC;
- known additional AGNs; and

- a big fraction of the known variable stars (including eruptive variable stars, novae and cataclysmic stars).

The OMC-IC includes both known variable stars as well as suspected variable stars. Special care was devoted to the compilation of extragalactic objects (AGN, QSO, starburst galaxies, narrow emission line galaxies, etc.) as they are potential highenergy emitters, and in many cases show significant variations of their optical flux. Indeed, Beckmann et al. (2009) compiled the second INTEGRAL AGN catalogue including optical information from OMC for 57 AGN.

In the compilation of optical sources, mostly available before the INTEGRAL launch, the SIMBAD database and the following catalogues were used:

1. Combined General Catalogue of Variable Stars, 4.1 (GCVS; Kholopov et al. 1998). It contains a catalogue of all known Galactic variable stars prior to 1997, a catalogue of extragalactic variable stars, a catalogue of supernovae and a catalogue of suspected variable stars not designated as variables prior to 1980 (published in The New Catalogue of Suspected Variable Stars, Kukarkin \& Kholopov 1982).

2. New Catalogue of Suspected Variable Stars. Supplement (Kazarovets et al. 1998). It contains 11206 stars suspected of variability which were not designated as variables prior to 1997.

3. The 74th Special Name-list of Variable Stars (Kazarovets et al. 1999). It contains 3157 variable stars whose variability was discovered by the HIPPARCos mission. All the stars satisfy the GCVS variability criteria.

4. The Hipparcos Catalogue (Perryman et al. 1997). The 11597 variable stars in the HIPPARCos catalogue were included.

5. Variable stars in the Tycho photometric observations. To search for variability among faint stars of the Tycho catalogue (Hoeg et al. 1997), Piquard et al. (2001) made a treatment that took into account truncated detections and censored measurements. The list contains 1091 stars suspected to be variable stars.

6. Quasars and Active Galactic Nuclei, 8th edn. (Veron-Cetty \& Veron 1998).

7. The Active Galactic Nuclei Catalogue (Padovani, priv. comm.). This catalogue includes 12021 quasars and active galaxies and is heavily based on the catalogue of Quasars and Active Galactic Nuclei, 7th edn. (Veron-Cetty \& Veron 1996). It also includes the BL Lac Catalogue (Padovani \& Giommi 1995) updated with BL Lac's discovered in 1996, and the radio galaxies in the $1 \mathrm{Jy}, \mathrm{S} 4$, and $\mathrm{S} 5$ radio catalogues. The AGN catalogue reports $V$ magnitudes almost for all of their objects. Nevertheless, in some cases $V$ magnitudes were derived from $B$ magnitudes by assuming a $(B-V)$ colour index.

8. Narrow Emission Line Galaxies (Kunth, priv. comm.). Compilation of 441 objects dominated by intense starburst activity. These objects show strong optical emission lines and/or very blue stellar continuum, both being tracers of young, massive stellar populations and therefore candidates for host supernovae.

9. Catalogue of Cataclysmic Variables (Downes et al. 2001). This catalogue contains 1134 sources that were included in the OMC-IC.

10. Candidates for classical T-Tauri stars. Classical T Tauri stars are good targets for OMC because they emit in the optical 

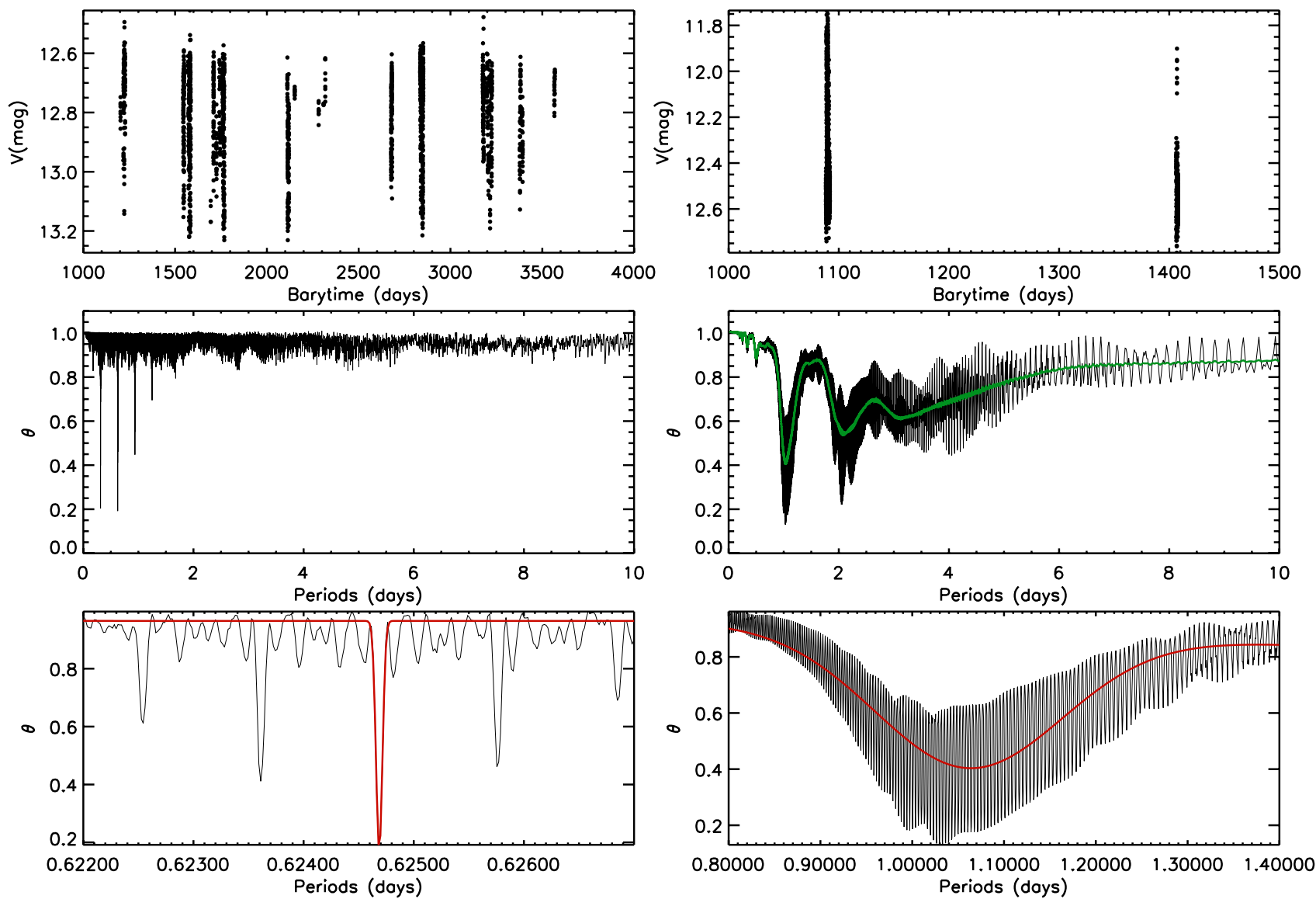

Fig. 1. Visual description of the process of period determination for two different cases: a standard situation (analysis of IOMC 0460000022) on the left and an example of aliasing (IOMC 5228000021) on the right. In both cases, the plots are: top: the light curve without folding. For the light curve affected by aliasing the large temporal gap can be appreciated. Middle: periodogram from 0 to 10 days. The peaks are much narrower in the standard case. In the aliasing example, the smoothed periodogram taking into account the aliasing effect (see text) is overplotted in green. Bottom: detail of the method used to determine the uncertainty of the period. In red, the continuum plus gaussian fit to the peak is plotted.

band in a periodic or non periodic way, but always have some type of variability related to their physical conditions. The amplitudes of the light curves are in the range 0.01-3 mag. Periods are often $\sim 1$ day, so the monitorization from a space platform should overcome the lack of observing them from the ground. 732 candidates for classical T-Tauri stars were selected (Caballero-García 2003) based on the segregation shown by these stars in a 2 MASS $(J-H)$ vs. $(H-K s)$ colour-colour diagram (Lee \& Chen 2002).

11. Candidates for cool dwarfs of $G-K-M$ spectral type. It is broadly known that cool dwarfs of G-K-M spectral type can be variable at a certain degree (mainly in the range 0.05-0.1 mag and not greater than $0.2 \mathrm{mag}$ ), showing very interesting properties of the behaviour of this kind of stars. A selection of 35101 candidates was made by CaballeroGarcía (2003) based on proper motions and the 2MASS $(J-H)$ and $(H-K \mathrm{~s})$ colours.

\section{Data analysis}

After the proprietary period of one year, all INTEGRAL data are open to the scientific community. At the moment of writing, INTEGRAL has been in orbit for more than nine years and the OMC database ${ }^{1}$; (Gutiérrez et al. 2004) now contains observations for more than 130000 objects, of which more than

\footnotetext{
1 http://sdc.cab.inta-csic.es/omc/
}

70000 have light curves with at least 50 photometric data points. The data included in this catalogue contain observations from the beginning of the mission until February 2010.

\subsection{Selection of the sources and cleaning of the light curves}

For this work, sources with more than 300 photometric points have been selected from the OMC database, in order to deal with light curves with enough points to study their variability and when possible, their periodicity. With the aim of improving the quality of the data some selection criteria have been applied to the individual photometric points.

First of all, we have checked if the coordinates in the OMCIC agree with the coordinates given in SIMBAD. The OMC database allows the user to select between two different centroid methods to extract the photometry, which corresponds to different options in the standard OMC Off-line Science Analysis (OSA) software distributed by the INTEGRAL Science Data Centre (ISDC; Courvoisier et al. 2003). If Source coordinates (default) is selected as the centroid method, the pipeline looks for the centroid allowing a maximum offset of 10 arcsec with respect to the given coordinates. If the centroiding algorithm fails by any reason, the extraction mask is nevertheless recentred right on the source coordinates as given in the OMC-IC. However, if brightest pixel is selected as the centroid method, the position of the extraction mask is re-centred on the brightest pixel within the central $5 \times 5$ pixel section. For the sources with 


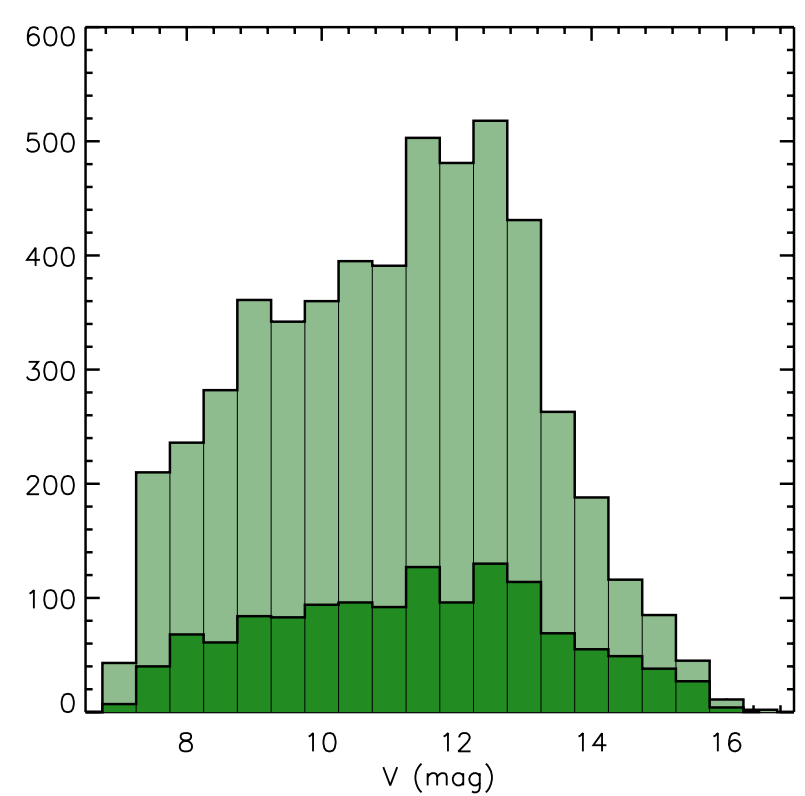

Fig. 2. Histogram of magnitudes, covering the range from 7.10 to 16.27 , with a peak of the distribution at around $12.3 \mathrm{mag}$. In this and subsequent histograms the fraction of sources for which we have determined a period in this work are represented with dark green.

a difference between the coordinates of the input catalogue and the coordinates given by SIMBAD greater than 10 arcsec, the brightest pixel method has been used. Moreover, in all the cases, those photometric points with a distance to the coordinates of SIMBAD greater than 10 arcsec have been rejected.

Secondly, we have looked for duplicated entries in the catalogue. There are sources in the input catalogue with different OMC identifiers, but with the same SIMBAD name or very close coordinates. These two situations have been taken into account and the duplicated sources have been removed from the list.

Typical OMC integration times are $10 \mathrm{~s}, 50 \mathrm{~s}$ and $200 \mathrm{~s}$, although at the begining of the mission shots of $10 \mathrm{~s}, 30 \mathrm{~s}$ and $100 \mathrm{~s}$ were obtained. Therefore, photometric points with different integration times can be found in the database. The longest integration times led to saturation of the brightest objects. For this reason, for each light curve we have rejected the photometric points that could be saturated. In general, all the photometric points within light curves where the median brightness is less than 7.1 were rejected. When the median brightness was less than 8.3, those photometric points with exposure times greater than $20 \mathrm{~s}$ were eliminated. If the median brightness was between 8.3 and 8.8 , only the photometric points with exposure times of 10 or $30 \mathrm{~s}$ were selected. For light curves with median brightness between 8.8 and 9.6 the exposures of 100 and $200 \mathrm{~s}$ were rejected. When the median brightness was between 9.6 and 10.3 , the photometric points with exposure times of $200 \mathrm{~s}$ were removed. And finally, for those sources with median brightness greater than 10.3 we considered that no problems of saturation should appear and all photometric points were considered.

In a similar way, some detection limits were defined to avoid photometric points with too low signal-to-noise ratio $(\mathrm{S} / \mathrm{N})$. For each light curve, those photometric points with exposure times of $10 \mathrm{~s}$ and a median brightness greater than 12, exposure times of $30 \mathrm{~s}$ and a median brightness greater than 13 and exposure times of $50 \mathrm{~s}$ and a median brightness greater than 14 have been removed.
Furthermore, measurements with low $\mathrm{S} / \mathrm{N}$ were excluded. A minimum $\mathrm{S} / \mathrm{N}$ of 10 was required for the shortest integrations $(10,30$ and $50 \mathrm{~s})$ while for the longest ones (100 s and $200 \mathrm{~s}) \mathrm{a}$ criteria of $S / N>3$ was imposed.

The observations were filtered from the effects of cosmic rays hits and some occasional detector artifacts. Each photometric point was compared with the 20 closest ones within 3 days (to avoid the timing gaps). For these 20 points, the standard deviation was computed and a 5-sigma rejection criteria was applied. We have checked manually that this algorithm removed most of the outliers. Finally, the photometric points that contained any hot pixel within their extracting mask of $3 \times 3$ pixels were removed.

In this first version of the OMC catalogue we have analysed only those objects with a given SIMBAD object type in the OMC-IC. Applying these filters we obtained 6071 sources with high-quality light curves to be studied. In the next version of the OMC catalogue we will include all objects in the OMC database complying with the previous requirements, independently of their assigned type, estimated to be around 25000.

\subsection{Detection of variability}

To detect which light curves showed variability we have used the $\chi^{2}$ goodness-of-fit test. This test compares a null hypothesis to an alternative hypothesis. If the value obtained for the test statistic is greater than a value corresponding to the chosen significance level of the $\chi^{2}$ distribution with $k=n-1$ degrees of freedom, being $\mathrm{n}$ the number of data points, then we reject the null hypothesis. We have used for each source the null hypothesis of being non-variable and having a constant magnitude equal to the mean. The alternative hypothesis is that the star is variable. Then, we calculated the $\chi^{2}$ and the significance $\alpha$. This significance gives the probability of being wrong when rejecting the null hypothesis (the source is constant). We have considered as variable those sources with $\alpha<0.05$ (a lower limit probability of being variable of $95 \%$ ). Following this criterion we have identified 5263 sources showing variability in their OMC light curves, which constitute our present catalogue. Though all 6071 sources were expected to be variable, we have not identified variability for 808 of them, according to the criteria we have applied. In some cases this can be due to the time span of the OMC observations, and in other cases the amplitude is likely below the OMC variability detection threshold used for this first version of the catalogue.

\subsection{Study of the periodicity}

To determine which sources are periodic and to derive their periods, an algorithm based on the phase dispersion minimization (PDM) technique (Stellingwerf 1978) has been developed. Given the variety of variability patterns present in our data we decided not to use the Lomb-Scargle (L-S) periodogram technique by Scargle (1982). This method evaluates the discrete Fourier transform for nonevenly sampled data. Since this is equivalent to assuming a prior sinusoidal light curve, we considered it not adequate for most eclipsing and non-sinusoidal light curves.

We explored a wide range of frequencies corresponding to periods from $0.8 \mathrm{~h}$ (a lower limit of the periods that can be detected by $\mathrm{OMC}$, considering the typical exposure times), up to half of the time range of the observations (the maximum period to have at least two cycles in the folded light curve). We first 


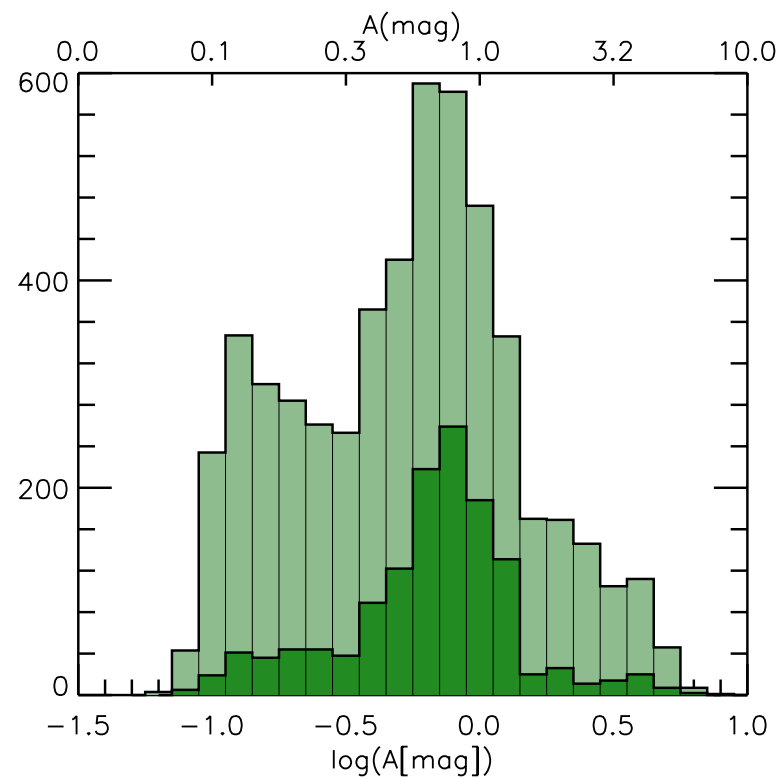

Fig. 3. Histogram of amplitudes (calculated as the difference between the magnitude at minimum and the magnitude at maximum of the light curve) with a peak of the distribution at around 0.84 mag. In dark green are represented the sources for which we have determined a period in this work.

used a frequency increment of $\frac{1}{3 B}$, being $B$ the total time baseline of the observations, and in a second iteration around the first trial period, we used a period increment of $2 P^{2} /(n B)$ (see Giménez \& García 1983), where $P$ is the trial period and $n$ is the number of points in the light curve. For each period, the timefolded light curve is divided into a series of bins and the variance of the amplitude within each bin with respect to a mean curve is computed. In our algorithm the number of bins is variable and it depends on the quality of each light curve. For each bin, the mean value is calculated and linear interpolations are done between the means to obtain one average curve for each folded light curve. The ratio between the sum of the bin variances and the overall variance of the data set is called $\Theta$, and the period that minimizes this parameter has been considered to be the best estimate.

With the values of the trial periods and their corresponding values of $\Theta$, we computed the periodograms. The uncertainty in the period was calculated by fitting a Gaussian to the peak corresponding to the minimum value of $\Theta$ in the periodogram. The 1-sigma error in the determination of the centroid of the Gaussian is provided for each source as the standard uncertainty of the period and corresponds to a confidence interval of $68.3 \%$ (see Fig. 1). For most of the sources the process above works fine, but in some cases aliasing becomes a problem. Aliasing occurs when a curve is observed with an insufficient sampling. For some sources in the catalogue there are temporal gaps in the observations much greater than the value of the period, and the time intervals of the different observations are not long enough to determine the period accurately. For a given estimated period $P_{i}$, if the temporal gap, $t_{\text {gap }}$ (the time when the source has not been observed), is such as $t_{\text {gap }}=N \times P_{i}$ (with $N$ big enough), there will be many significant peaks in the periodogram, close to the deepest one, corresponding to that values of the period that make $t_{\text {gap }}=(N+1) \times P_{i-1}$ or $t_{\text {gap }}=(N-1) \times P_{i+1}$ (see Fig. 1 , right panel). In these cases we have smoothed the periodogram with a window size wider than the distance between these false peaks. This

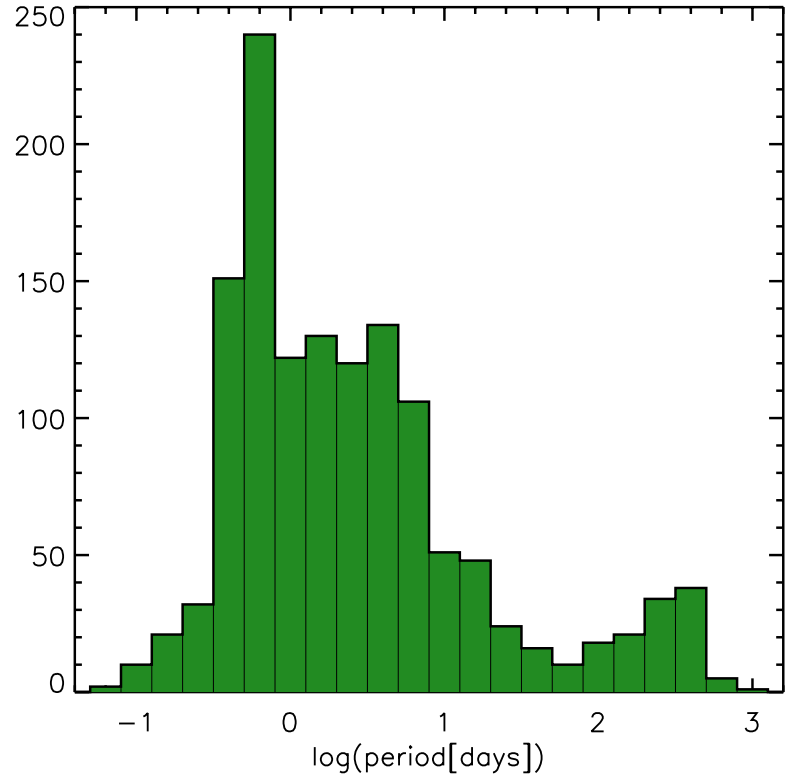

Fig. 4. Histogram of the periods derived. Typical values vary between a few hours and 10 days, with a peak of frequency at around $15 \mathrm{~h}$.

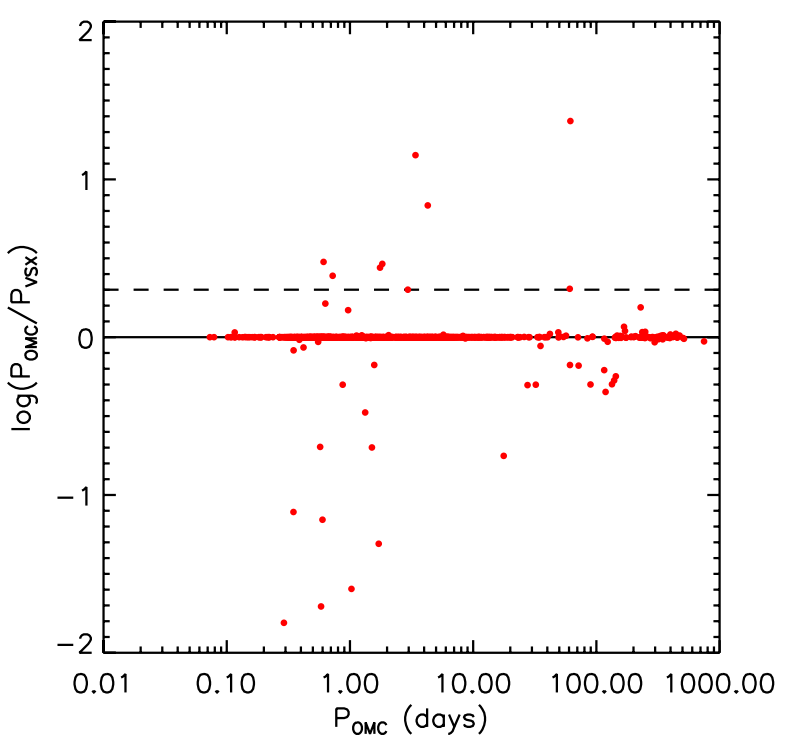

Fig. 5. Comparison of periods derived from OMC data (this work) with the values compiled by the VSX. The solid line represents $P_{\mathrm{OMC}}=P_{\mathrm{VSX}}$ while the dashed line marks the condition $P_{\mathrm{OMC}}=2 P_{\mathrm{VSX}}$.

smoothed curve is used to determine the limit of significance of the peak and the half width of this peak is used as initial value of sigma in the Gaussian fit. Finally, a visual inspection of each folded light curve was performed to identify and correct potential problems due to ambiguities in the results, such as deriving the half or double period in some eclipsing binaries or pulsating stars with symmetrical light curves.

The method used in this work yields only the primary periods. To obtain second or multiple periods, if present, a manual analysis of each source is required and this is out of the scope of this paper.

We show in Fig. A.5 the light curve of Her X-1, one of the brightest and most studied persistent X-ray binary pulsars. The system displays a great variety of phenomena at different timescales, including pulsations at $1.24 \mathrm{~s}$, eclipses at the orbital 
Table 1. Contents of the OMC-VAR catalogue.

\begin{tabular}{lll}
\hline \hline Field & Source & Description \\
\hline IOMC & OMC & OMC Identifier \\
SIMBAD name & SIMBAD & SIMBAD Basic Identifier \\
RA & SIMBAD & Right ascension in sexagesimal units (J2000) \\
DE & SIMBAD & Declination in sexagesimal units (J2000) \\
RAdeg & SIMBAD & Right ascension in degrees (J2000) \\
DEdeg & SIMBAD & Declination (J2000) in degrees (J2000) \\
otype & SIMBAD & SIMBAD object type \\
sptype & SIMBAD & SIMBAD spectral type \\
vartype & VSX & VSX variability type \\
vargroup & VSX, SIMBAD, others & General variability group \\
$V$ mag & OMC & Median $V$ magnitude \\
magerr & OMC & Mean of the magnitude error \\
magMax & OMC & Magnitude at maximum brightness \\
magMin & OMC & Magnitude at minimum brightness \\
per & OMC & Period of variability in days \\
errper & OMC & Uncertainty of the period in days \\
timebase & OMC & Time base of the observations in days \\
tagcont & UCAC3, NOMAD1, SIMBAD, VSX & Tag of contamination \\
\hline
\end{tabular}

Notes. Accessible at http://sdc. cab.inta-csic.es/omc/var/ and at CDS services.

period of 1.7 days, and a 35 day cycle in the X-ray intensity (Leahy \& Dupuis 2010). Despite the large gap between the two epochs at which this object has been observed by OMC, our method retrieves properly the orbital period. Nevertheless, the length of the gap, compared to the short orbital period, implies that the period has been derived with a rather low accuracy.

\subsection{Photometric contamination by nearby stars}

For the data analysed in this study, fluxes and magnitudes were derived from a photometric aperture of $3 \times 3$ pixels $(1$ pixel $=$ 17 '.504), slightly circularized, i.e., removing $\frac{1}{4}$ of a pixel from each corner (standard output from OSA). Therefore the computed values include the contributions by any other source inside the photometric aperture. We flagged in a column of the catalogue those sources that might be affected by this kind of photometric contamination. Due to the variable nature of the sources in the catalogue and the difficulty to accurately estimate the contamination by the nearby and background stars, we developed three different methods. The first one was to take into account the photometric information of the sources close to the extraction aperture present in the UCAC3 (Zacharias et al. 2010) and NOMAD1 (Zacharias et al. 2004) catalogues. The second one was to compare the $V$ magnitude provided by SIMBAD (for variable sources it is usually the magnitude at maximum brightness) with the lowest magnitude we measure for each source, and the third one was to compare the median magnitude in VSX with the median magnitude we measure. We considered a source significantly contaminated when the measured flux was a factor of 2 higher than expected. This is equivalent to a difference between expected and observed magnitude of more than $0.75 \mathrm{mag}$. While this criterion is not very restrictive in terms of photometric accuracy, it allows to study variability and search for periodicity for objects with a significant degree of contamination. We want to stress nevertheless that this procedure has the caveat that in some cases the variability/periodicity found might be originated by the contaminating object(s), and not by the target source. One of the objects mostly affected by blending is HH Nor, shown in Fig. A.6, whose variability pattern is completely dominated by the variability of the brighter, nearby object VSX J154329.4-515037, which is indeed a variable RR Lyr star with a much shorter period. Being located at just $12^{\prime \prime}$, a fraction of an OMC pixel, the intrinsic variability of this contaminating star completely hides the variability of the target source, HH Nor.

The results of these three methods are coded in the column of contamination with three numbers (a reference to each method) where 1 means that the source is not contaminated (under the conditions defined above), 2 means that it is contaminated and 3 means no photometric information has been found for that source. Because of the inherent difficulties of estimating the contamination of variable sources, this flag is for guidance only and to study individual sources a more exhaustive analysis of the sky field around each source is recommended.

\section{Contents of the OMC-VAR catalogue}

Table 1 describes the contents of the OMC-VAR catalogue, which is fully accessible online. In this first release we provide information about the variability of 5263 objects distributed all over the sky (see Fig. 6). The majority of the objects are located in the Galactic plane and Galactic bulge, as well as on specific areas of the sky. This distribution reflects the integration time density maps of INTEGRAL, driven by the location of the most interesting high-energy sources.

For each source we include the median value of the $V$ magnitude, the mean of the photometric errors of the points and the magnitudes at maximum and minimum brightness, estimated as the 2nd and 98th percentiles, respectively, of the points in the light curve. For those sources classified as periodic or probably periodic, we provide the best estimate of the period and its calculated uncertainty.

The values of the median magnitudes go from 7.10 mag to 16.27 mag. In Fig. 2 we show the histogram of the median magnitudes, showing a peak at around $12.3 \mathrm{mag}$. A similar plot for the amplitudes (calculated as the difference between the magnitude at minimum and the magnitude at maximum of the light curve) is shown in Fig. 3. The amplitudes go from 0.06 mag to $7.41 \mathrm{mag}$ with a mean value of $0.84 \mathrm{mag}$. 


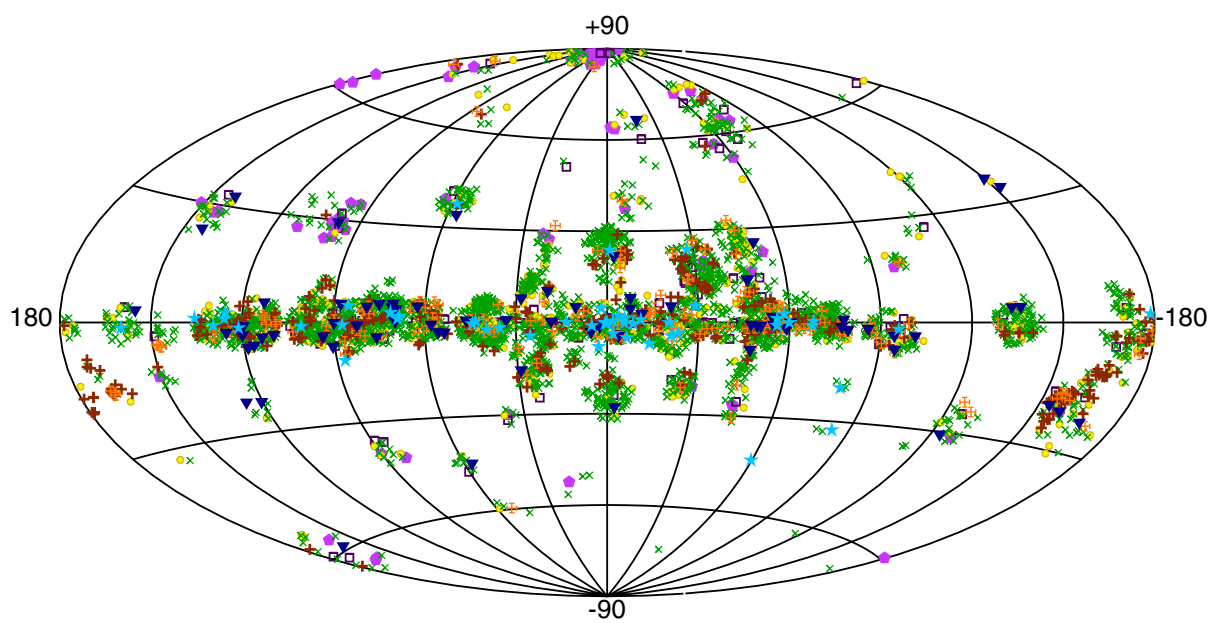

Fig. 6. Distribution in galactic coordinates of all the sources in the catalogue. The green crosses represent pulsating stars, the red filled points correspond to eclipsing binaries, the brown plusses are eruptive stars, the orange complex plusses represent rotating stars, the inverted dark blue filled triangles represent cataclysmic variables, the light blue filled stars are X-ray binaries, the yellow filled points correspond to objects simply classified as variable stars, the purple filled pentagons represent extragalactic objects and the empty purple squares are other types of objects.

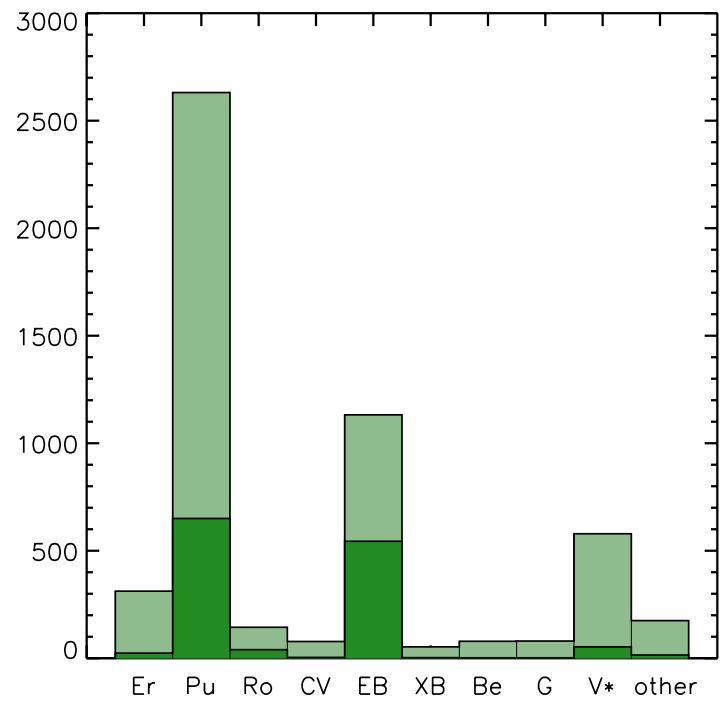

Fig. 7. Histogram of the different groups of variability types present in the catalogue. There are a big number of objects which are unclassified, or just classified as variable objects.

The time base for each light curve is also provided. These time bases have a minimum value of $0.69 \mathrm{~d}$, a maximum value of $2815.19 \mathrm{~d}$ and a mean value of $1862.0 \mathrm{~d}$.

We have determined periods for 1337 sources, out of the 5263 variable objects compiled in the catalogue. The distribution of periods is shown in Fig. 4. Typical values vary between a few hours and 10 days, with a peak of frequency at around $15 \mathrm{~h}$. Some sources present periods of up to some hundreds of days, but there are not many of them given the time span and distribution of the observations. The VSX database also provides information about the periodicity of the sources and we have used this information to compare with our results. We have derived periods for 175 objects whose periodicity was unknown and in many other cases, we have improved the results with respect to those found in the VSX database, as can be seen in Appendix A.

In Fig. 5 we compare the periods derived from OMC data in this work with the values compiled in the VSX, when both were available. For the large majority of objects the values are consistent, though we want to stress that in many cases, even a very small improvement on the determination of the period is enough to yield a satisfactory folding of the light curve (see the examples in Appendix A). A dashed line marks the points for which $P_{\mathrm{OMC}}=2 \times P_{\mathrm{VSX}}$, to identify those cases of binaries with eclipses of similar shape that could have been misidentified in the VSX. In the rest of the cases we have verified that the period given in the VSX was not consistent with the OMC light curves. This happened sometimes for systems with multiple periods due to different variability mechanisms like rotation, orbital, X-rays, etc. One example is Her X-1 for which, as we mentioned in Sect. 3.3, the period given by VSX does not correspond to the orbital one to which OMC is more sensitive (see Fig. A.5).

\subsection{Classification}

A big variety of variable objects have been observed by OMC and are present in this version of the catalogue. In Fig. 6 the distribution in galactic coordinates of the different kind of objects is plotted. In order to do a statistical study of the type of objects in the catalogue we have used the information available in the literature. When available, the object classification has been extracted from the VSX. Otherwise, the type of variability has been taken from the SIMBAD database. According to the above classifications we have defined different variability groups and have assigned each source to one of them. In the case of pulsating stars we have defined 10 additional subgroups, and in the case of eclipsing binaries we have included 4 additional subgroups, based only on the shape of the light curve:

- 2631 pulsating stars $(\mathrm{Pu})$, including

- 297 Cepheids (Ce);

- 261 RR Lyrae (RR) pulsators;

- $37 \delta$ Scuti pulsators ( $\delta$ Sct);

- 32 RV Tau stars (RV);

- $28 \beta$ Cephei stars ( $\beta$ Cep);

- 26 SPB pulsators (SPB);

- 680 Mira type stars (M);

- 591 semi-regular stars (SR);

- 630 slow pulsators (L);

- 49 simply classified as pulsators (other). 

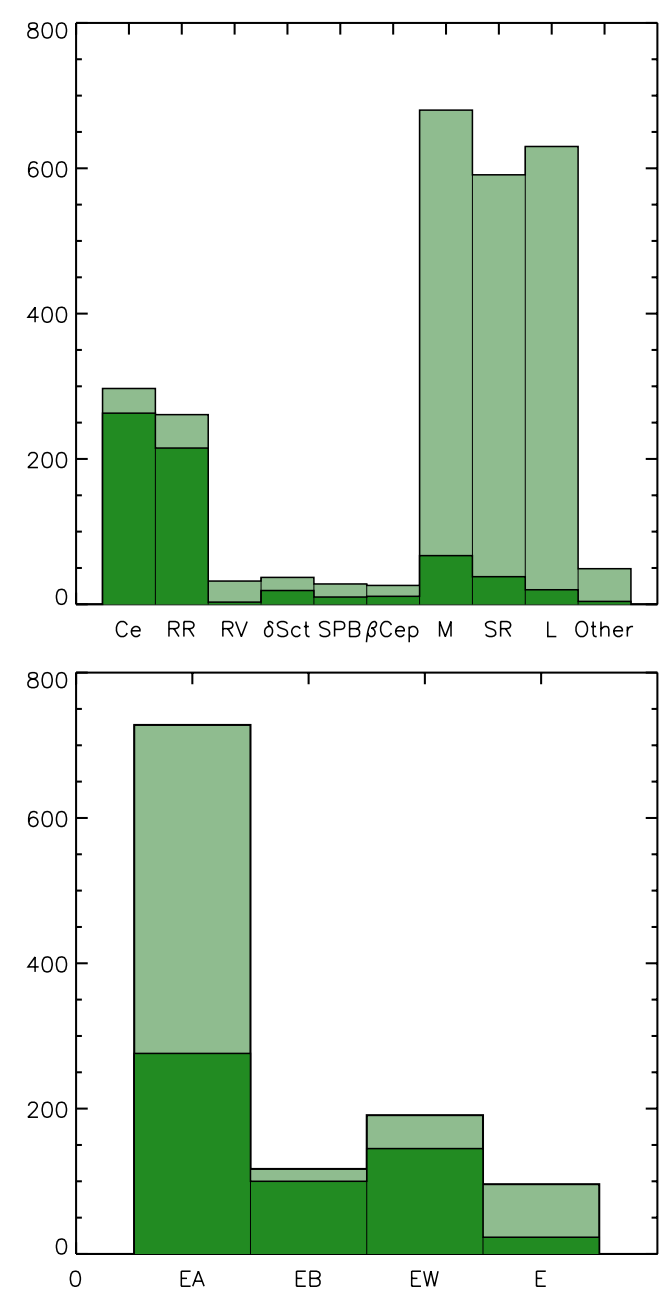

Fig. 8. Histogram of the subgroups of variability types present in the catalogue. Top: histogram of the different kinds of pulsating stars. Bottom: histogram of the subtypes of eclipsing binary stars.

- 1132 eclipsing binaries (EB), including

- 728 Algol ( $\beta$ Persei)-type eclipsing systems (EA);

- $191 \beta$ Lyrae-type eclipsing systems (EB);

- 117 W Ursae Majoris-type eclipsing variables (EW);

- 96 simply classified as eclipsing (E)

- 53 X-ray binaries $(\mathrm{XB})$;

- 78 cataclysmic variables $(\mathrm{CV})$;

- 312 eruptive stars (Er);

- 144 rotating stars (Ro);

- 79 Be stars $(\mathrm{Be})$;

- 579 stars just classified as variable $\left(\mathrm{V}^{*}\right)$;

- 80 extragalactic sources $(\mathrm{G})$; and

- 162 objects of other types.

We show in Fig. 7 the histogram corresponding to the different groups, clearly dominated by the groups of pulsating stars and eclipsing binaries. In Fig. 8 we detail the histograms for the subgroups of pulsating stars and eclipsing binary systems, the latter dominated by Algol-type systems.

Some examples of the kind of objects that can be found in the catalogue are shown in Appendix A, where we have plotted some representative charts including the DSS image around the target, and their light curves, unfolded and folded with the periods we have derived and/or with the catalogued ones.

\section{Summary}

We have analysed the optical photometric light curves, covering the time from the beginning of the mission to February 2010, of the 6071 objects present in the OMC database that satisfy the criteria explained in Sect. 3, with the aim of characterizing their expected variability and potential periodicity.

In this first version of the INTEGRAL-OMC catalogue of optically variable sources we have detected variability for 5263 objects, with median brightnesss in the range from 7.1 to 16.27 in $V$.

For 1337 of them we have been able to derive a period with enough significance. The periods found vary from a few hours to some hundreds of days, with a peak in the histogram of typical periods around $15 \mathrm{~h}$. We inspected visually the light curves to check the validity of the estimated periods, rejecting those false positives yielded by the PDM algorithm.

For each source in the catalogue we include the median value of the $V$ magnitude, the mean of the photometric errors of the points and the minimum and maximum value of $V$, estimated as the 98th and 2nd percentiles, respectively, of the points in the light curve. For those sources classified as periodic or probably periodic, we provide the best estimate of the period and its calculated uncertainty. We have also included a set of tags to identify those sources whose photometry can be significantly affected by nearby stars, as well as some ancillary data.

This catalogue contains data useful for the study of several classes of variable stars: eclipsing binaries, pulsating stars, active stars, cataclysmic variables, X-ray binaries, etc. The most frequent objects in the present compilation are pulsating stars and eclipsing binary systems, according to the classification provided by the VSX catalogue and SIMBAD. A deeper study of some of the sources presented here is being carried out and will be presented in forthcoming papers.

The next version of the INTEGRAL-OMC catalogue of optically variable sources will include all other objects in the OMC database complying with the same photometric requirements, estimated to be around 25000 .

Acknowledgements. The development, operation and exploitation of OMC have been funded by Spanish MICINN under grants AYA2008-03467 and AYA2011-24780 and previous ones. INTEGRAL is an ESA project funded by ESA member states (especially the PI countries: Denmark, France, Germany, Italy, Spain, Switzerland), Czech Republic, Poland, and with the participation of Russia and the USA. OMC was also funded by Ireland, United Kingdom, Belgium and the Czech Republic. This research has made use of data from the OMC Archive at CAB (INTA-CSIC), pre-processed by ISDC, and of the SIMBAD database, operated at CDS, Strasbourg, France.

\section{Appendix A: Charts provided for each object in the catalogue}

Some illustrative examples of the charts provided with the catalogue for different kinds of objects. The information about type of object, variability type and spectral type on the top of each plot comes from Simbad and VSX. In all cases we show the DSS red image of the field of view in the top left panel (red circles represent the OMC photometric aperture), and the OMC $V$-band light curve without folding in the top right one. The time is the Barycentric INTEGRAL Julian Date expressed in Barycentric Dynamical Time (TDB). To convert this time to Barycentric Julian Date expressed in TDB, you have to add $2451544.5 \mathrm{~d}$. Similar charts and the complete light curves in machine readable format for all the sources contained in the OMC-VAR Catalogue can be retrieved from http://sdc. cab.inta-csic.es/omc/. 
IOMC $7864000021 \quad \mathrm{~V} *$ IT Nor

O Type: Al* Var Type: EB/KE SP Type: B9.5III
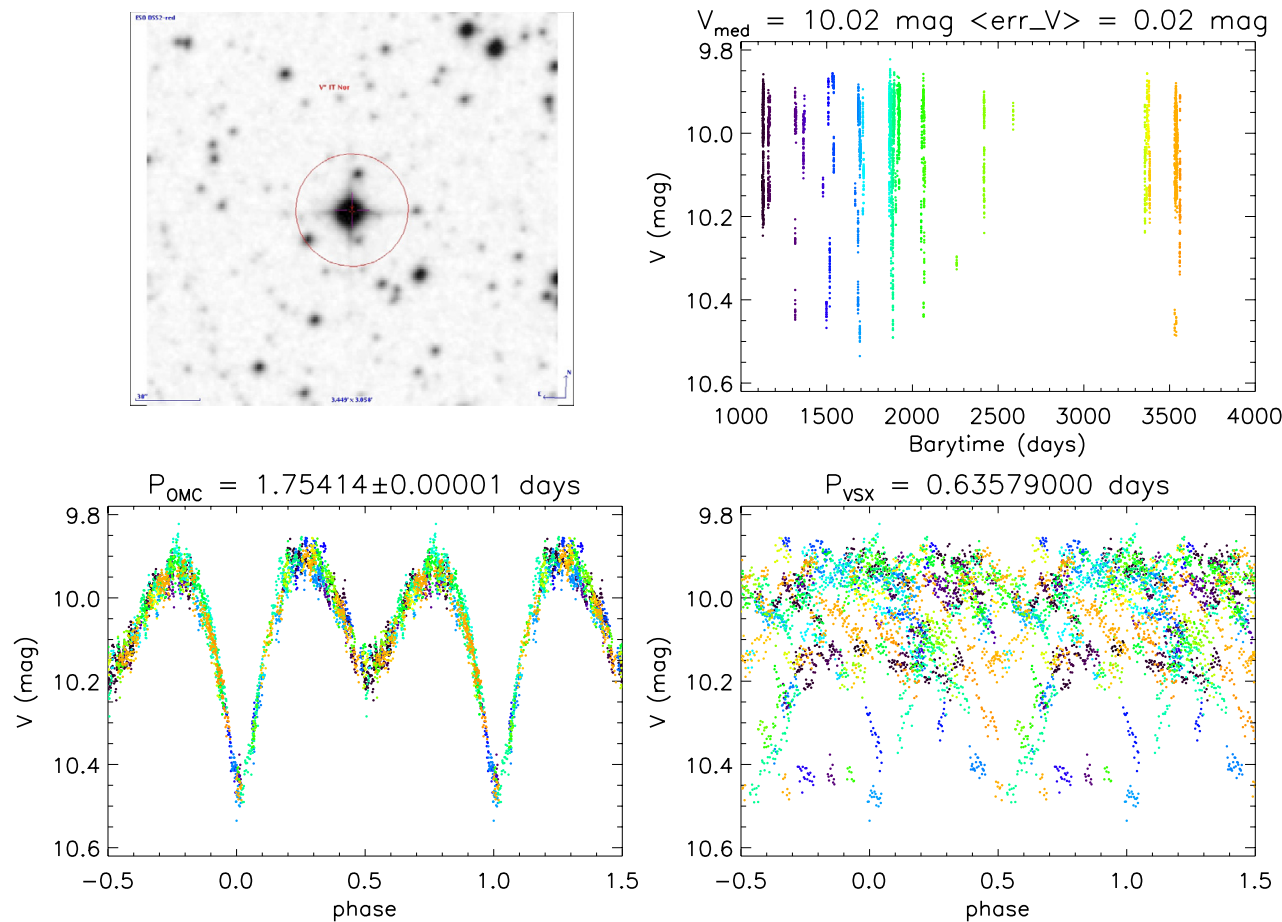

Fig. A.1. IT Nor, an Algol type eclipsing binary. When not indicated otherwise, we show in the different charts the OMC light curve folded with the period estimated in this work (bottom left), and the OMC light curve folded with the period given by the VSX catalogue (bottom right panel). In this case the period derived from the OMC light curve improves significantly the value provided by the VSX and yields a satisfactory folding.

IOMC $1991000096 \quad V * S$ Com

O Type: RR* Var Type: RRAB SP Type: F4
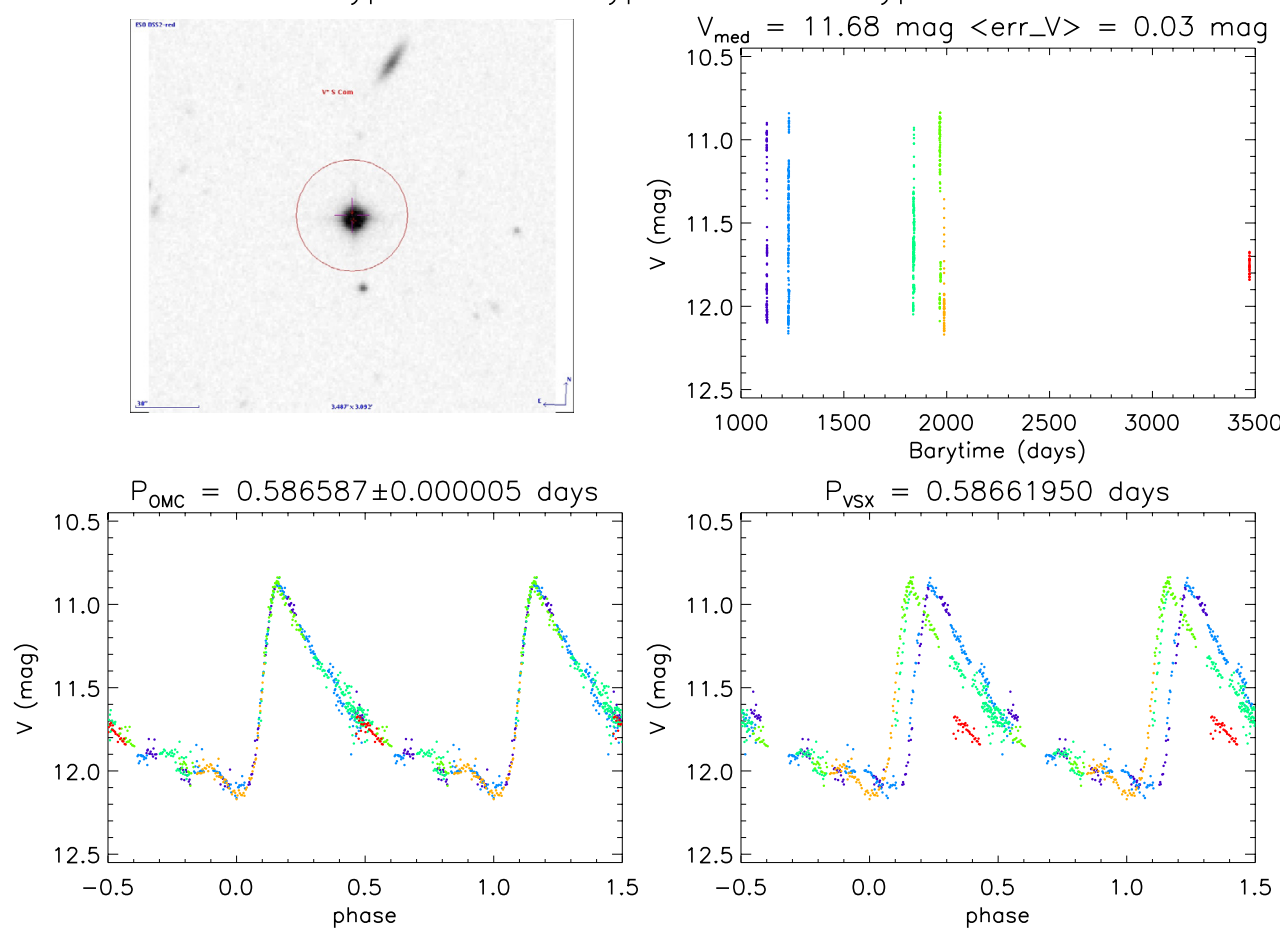

Fig. A.2. For some objects, like the RR Lyr star S Com, a very small increase in the accuracy of the period is enough to get a perfect folding of the light curve. 
IOMC $1042000027 \quad \mathrm{~V} * \mathrm{~V} 600 \mathrm{Aql}$

O Type: cC* Var Type: DCEP SP Type: F8v
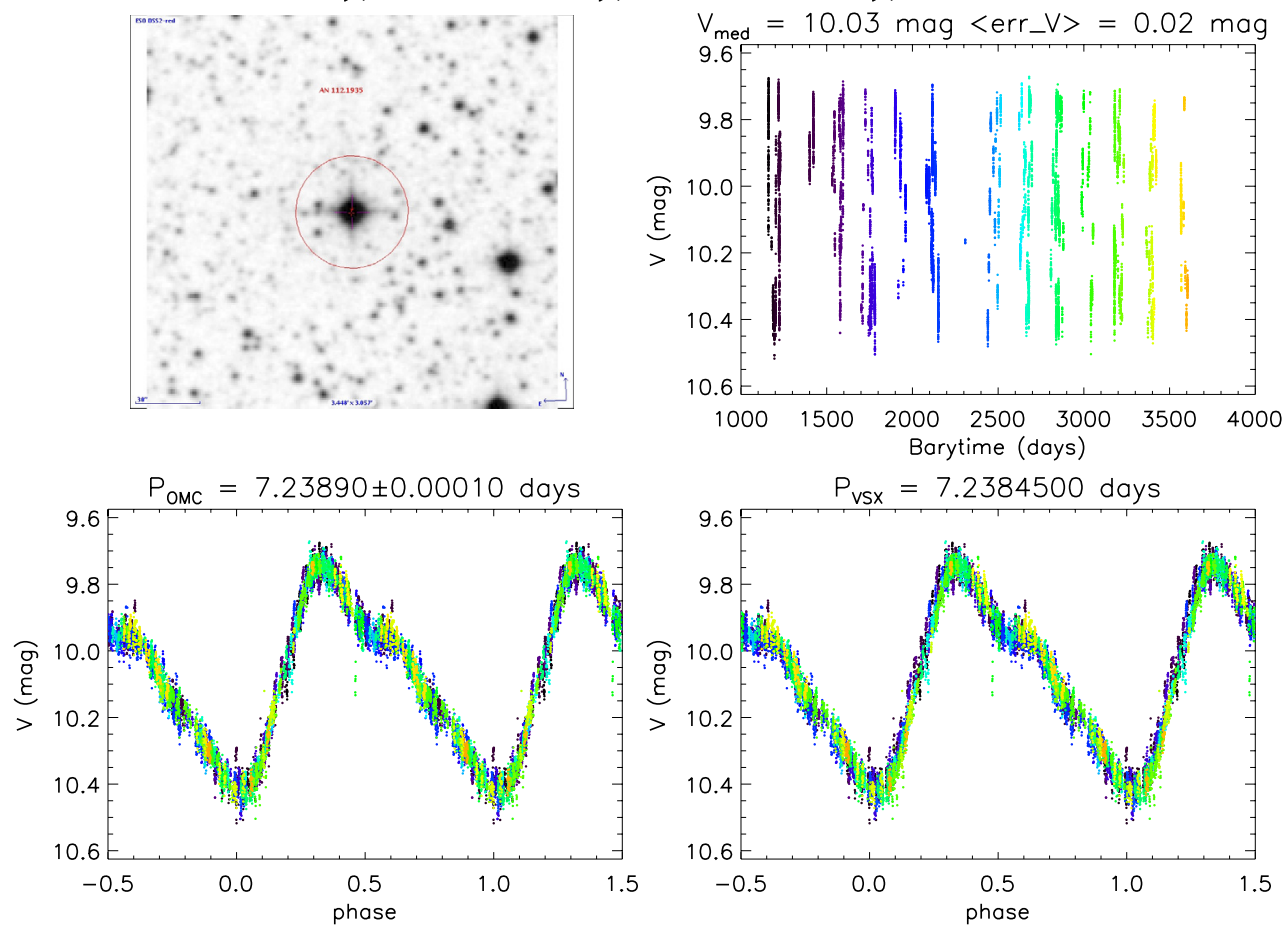

Fig. A.3. For a large fraction of objects, the period derived from OMC data and that compiled by VSX yield similar results when folding the light curve.

IOMC $0122000035 \quad V * V 651$ Ori

O Type: $\vee *$ Var Type: RR: SP Type:
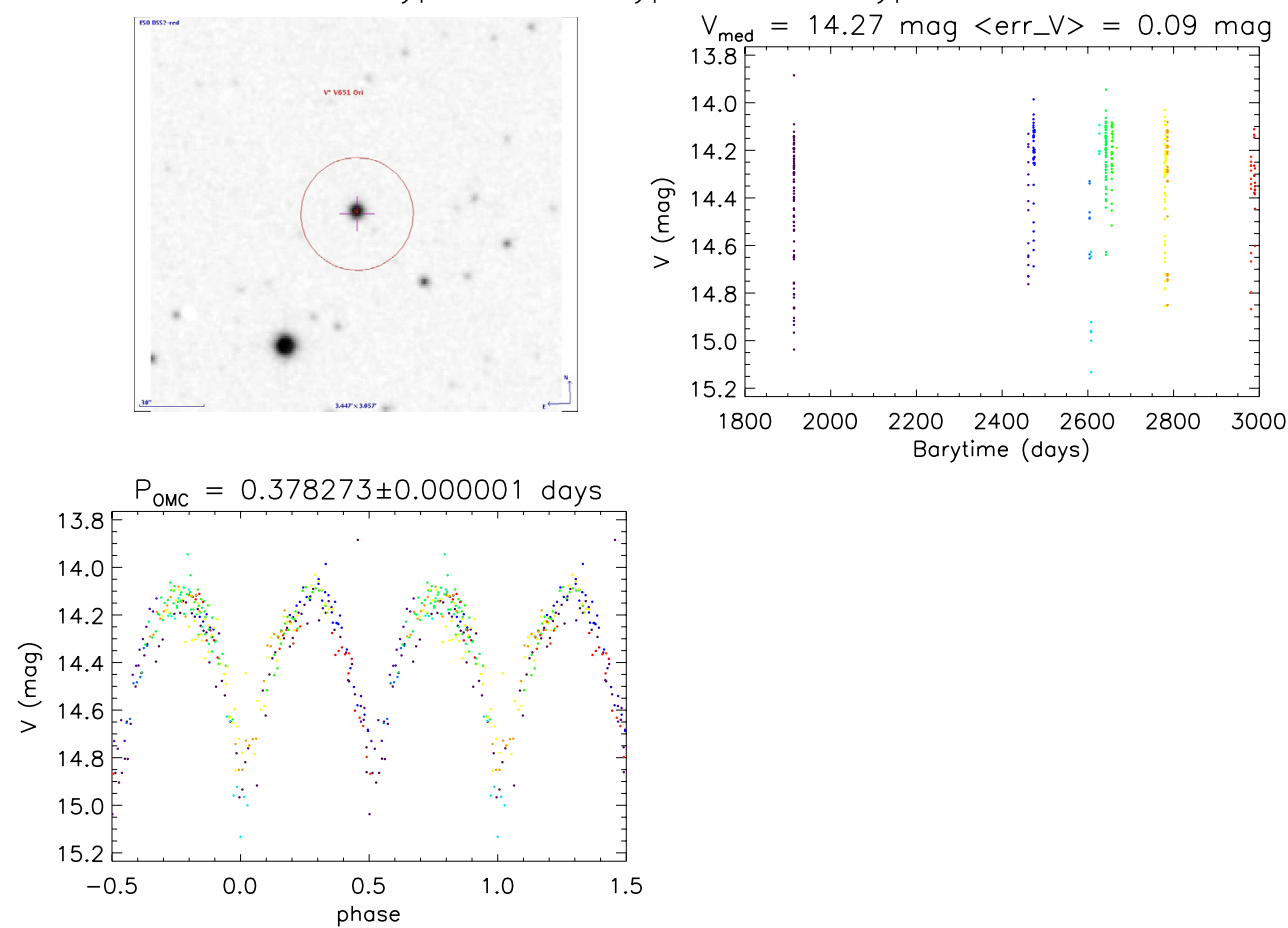

Fig. A.4. New period determination from this work for V651 Ori. Moreover, the variability classification in VSX should be corrected from RR Lyrae to eclipsing binary, according to our light curve. 
J. Alfonso-Garzón et al.: The first INTEGRAL-OMC catalogue of optically variable sources

IOMC $2598000079 \quad \mathrm{~V} * \mathrm{HZ}$ Her

O Type: LXB Var Type: LMXB:/XPR+E SP Type: DA
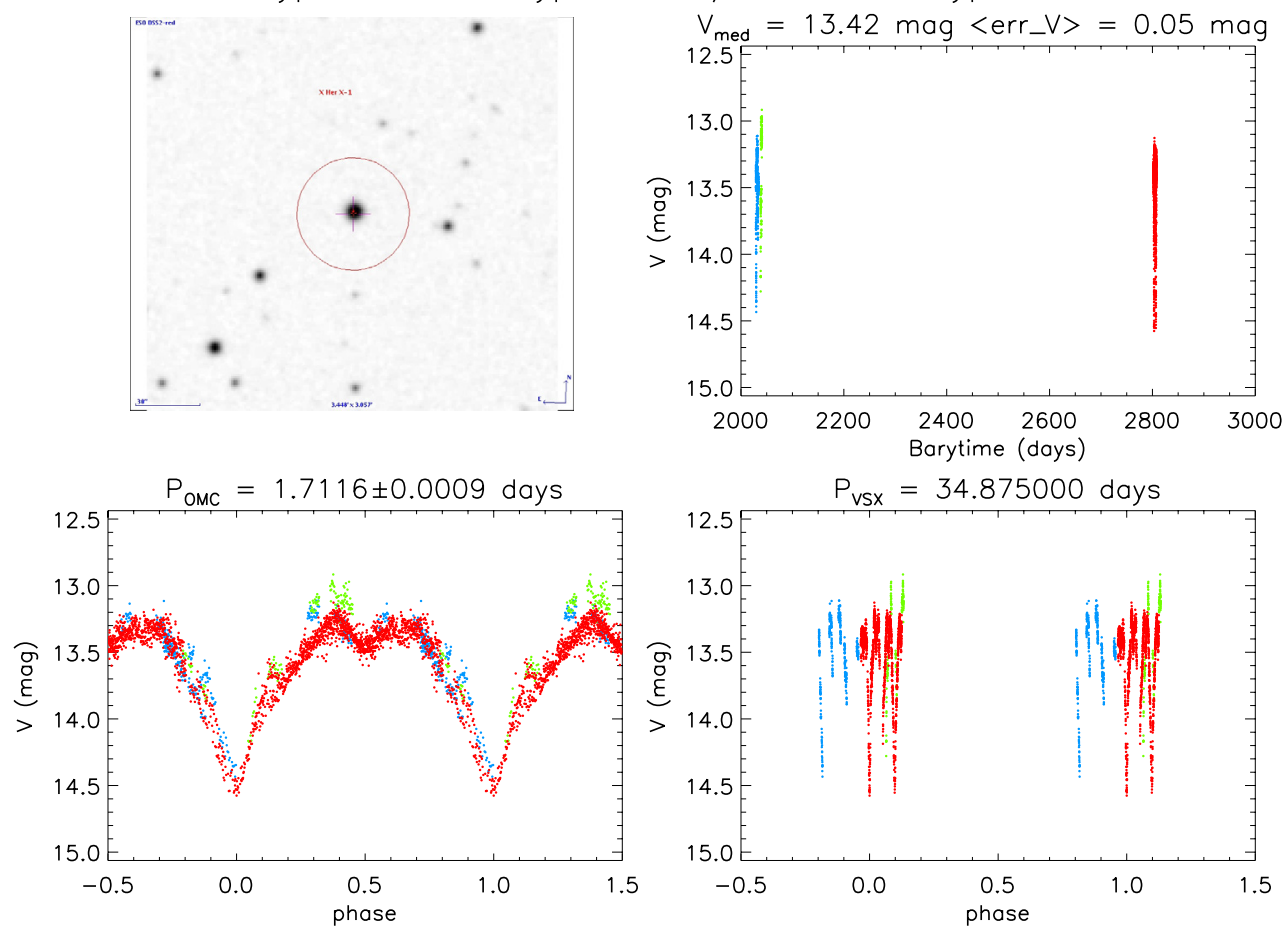

Fig. A.5. In the case of Her X-1, the VSX period corresponds to the 35 day cycle in the X-ray intensity, while the OMC optical light curve is dominated by the orbital eclipses.

IOMC $8308000051 \quad \mathrm{~V} * \mathrm{HH}$ Nor

O Type: Al* Var Type: EA/DS SP Type: FO
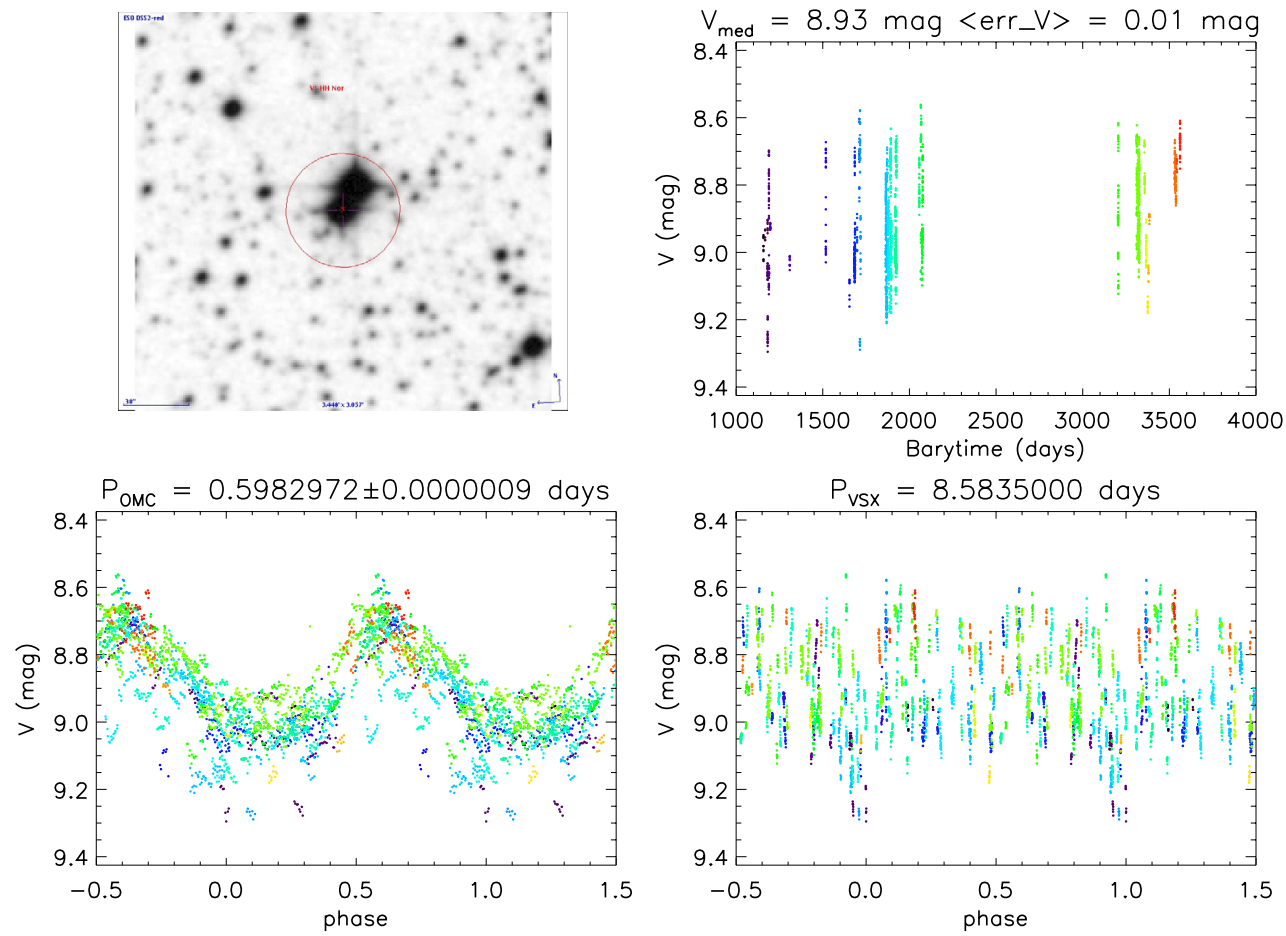

Fig. A.6. The photometry of the intended target, HH Nor, is heavily dominated by the brighter, nearby object VSX J154329.4-515037, which is indeed a variable RR Lyr star (RRAB) with a period of 0.59 days. In fact, HH Nor is an eclipsing binary with mean $V$ magnitude of 10.9 and a period of 8.58 days. 


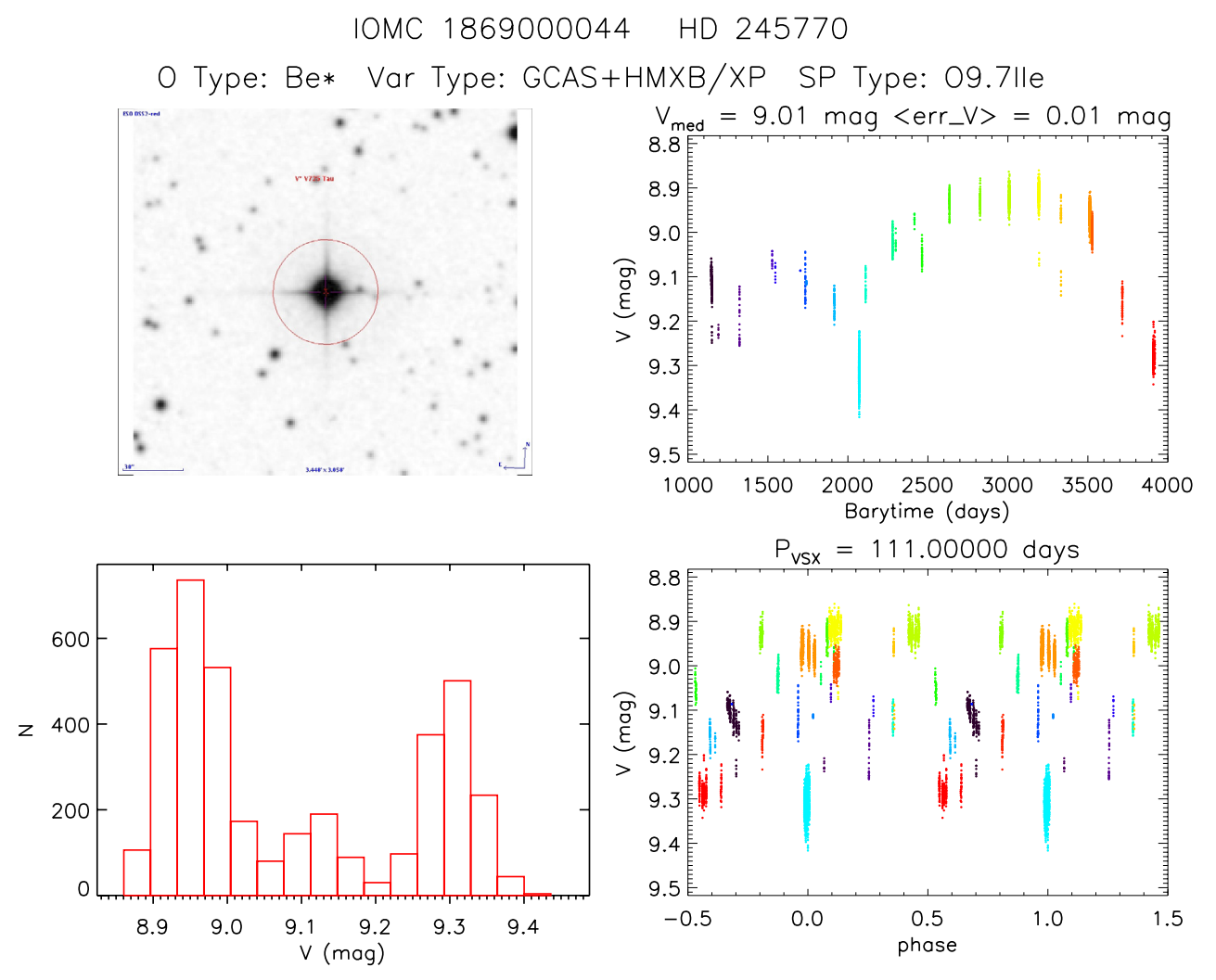

Fig. A.7. In the light curve of this high-mass X-Ray binary we see that a long term variation during the nearly 3000 days of monitoring dominates over the orbital period given by VSX. When there is no determination of the period from this work, the chart of the corresponding object includes a histogram of the observed magnitudes. The shape of these histograms helps to understand the variability pattern of the different objects.

IOMC $4368000068 \quad 8 \mathrm{C} 0716+714$

O Type: BLL Var Type: BLLAC SP Type:
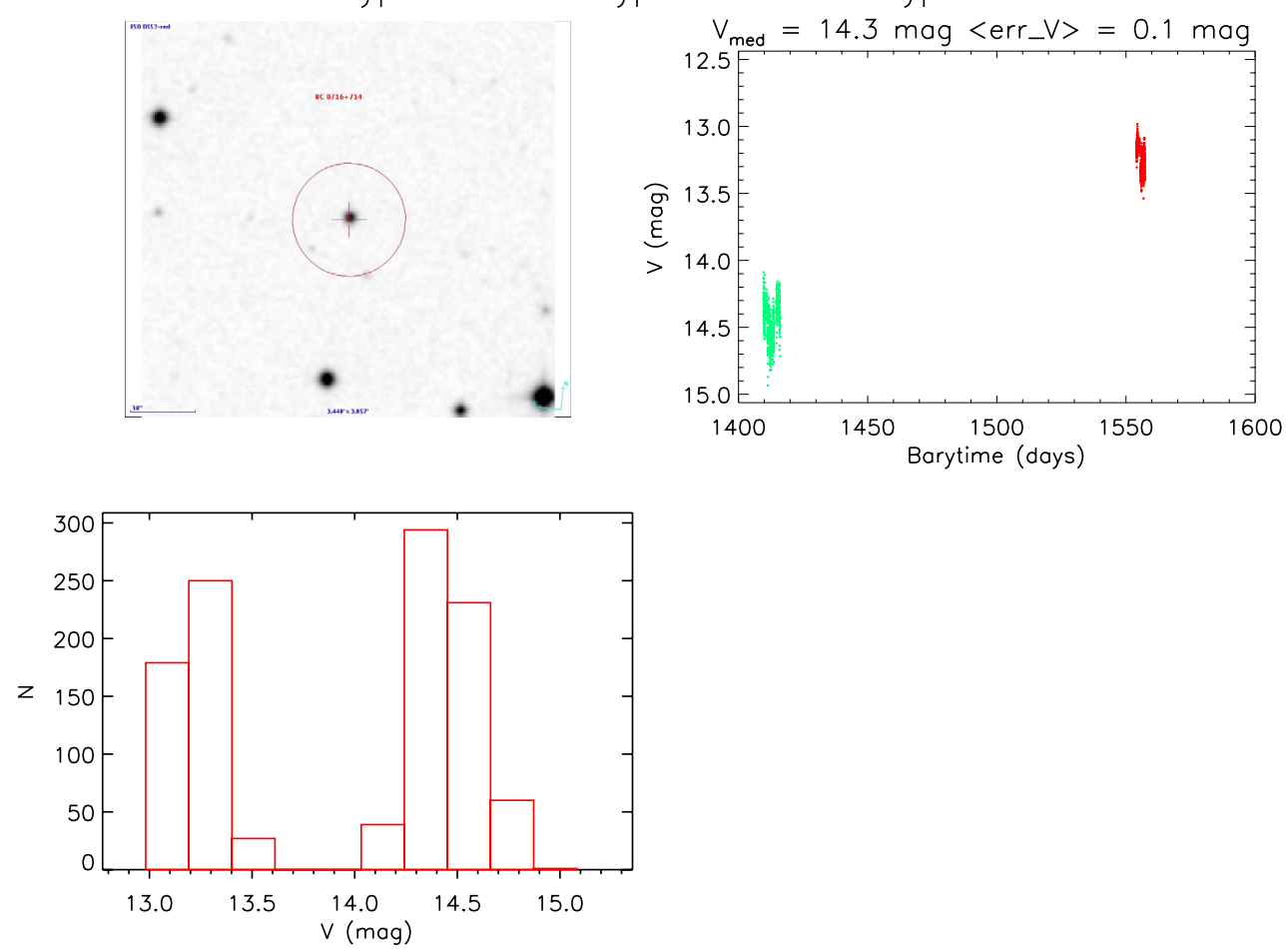

Fig. A.8. The OMC light curve of this blazar shows two different states, separated by around 150 days. They can be clearly observed in the magnitude histogram. 


\section{References}

Auvergne, M., Bodin, P., Boisnard, L., et al. 2009, A\&A, 506, 411 Beckmann, V., Soldi, S., Ricci, C., et al. 2009, A\&A, 505, 417 Bruntt, H., \& Buzasi, D. L. 2006, Mem. Soc. Astron. It., 77, 278

Caballero-García, M. D. 2003, Master's Thesis, Universitat de Barcelona Courvoisier, T. J. L., Walter, R., Beckmann, V., et al. 2003, A\&A, 411, L53 Domingo, A., Caballero, M. D., Figueras, F., et al. 2003, A\&A, 411, L281 Downes, R. A., Webbink, R. F., Shara, M. M., et al. 2001, PASP, 113, 764 Giménez, A., \& García, J. M. 1983, Rev. Real Acad. Cienc. Exact., 1, 297

Gutiérrez, R., Solano, E., Domingo, A., \& García, J. 2004, in Astronomical Data Analysis Software and Systems (ADASS) XIII, eds. D. E. F. Ochsenbein, M. G. Allen, ASP Conf. Ser, 314, 153

Hoeg, E., Bässgen, G., Bastian, U., et al. 1997, A\&A, 323, L57

Kazarovets, A. V., Samus, N., Durlevich, O. V., et al. 1999, Informational Bulletin on Variable Stars, 4659, 1

Kazarovets, V., Samus, N., \& Durlevich, O. V. 1998, Informational Bulletin on Variable Stars, 4655, 1

Kholopov, P. N., Samus, N. N., Frolov, M. S., et al. 1998, in Combined General Catalogue of Variable Stars, 4.1 Ed (II/214A), 0

Koch, D. G., Borucki, W. J., Basri, G., et al. 2010, ApJ, 713, L79

Kukarkin, B. V., \& Kholopov, P. N. 1982, New catalogue of suspected variable stars (Moscow: Publication Office Nauka)

Leahy, D. A., \& Dupuis, J. 2010, ApJ, 715, 897

Lee, H. T., \& Chen, W. P. 2002, in 8th Asian-Pacific Regional Meeting, Vol. II, eds. S. Ikeuchi, J. Hearnshaw, \& T. Hanawa, 161

Lund, N., Budtz-Jørgensen, C., Westergaard, N. J., et al. 2003, A\&A, 411, L23

Mas-Hesse, J. M., Giménez, A., Culhane, J. L., et al. 2003, A\&A, 411, L261
Nichols, J. S., Henden, A. A., Huenemoerder, D. P., et al. 2010, VizieR Online Data Catalog, 218, 80473

Padovani, P., \& Giommi, P. 1995, MNRAS, 277, 1477

Perryman, M. A. C., Lindegren, L., Kovalevsky, J., et al. 1997, A\&A, 323, L49

Piquard, S., Halbwachs, J. L., Fabricius, C., et al. 2001, A\&A, 373, 576

Pojmanski, G. 1997, Acta Astron., 47, 467

Samus, N. N., Durlevich, O. V., et al. 2012, GCVS database, CDS B/gcvs

Scargle, J. D. 1982, ApJ, 263, 835

Stellingwerf, R. F. 1978, ApJ, 224, 953

Ubertini, P., Lebrun, F., Di Cocco, G., et al. 2003, A\&A, 411, L131

Udalski, A., Szymanski, M., Kaluzny, J., Kubiak, M., \& Mateo, M. 1992, Acta Astron., 42, 253

Vedrenne, G., Roques, J. P., Schönfelder, V., et al. 2003, A\&A, 411, L63

Veron-Cetty, M. P., \& Veron, P. 1996, A Catalogue of quasars and active nuclei: European Southern Observatory (ESO), 7th edn.

Veron-Cetty, M. P., \& Veron, P. 1998, A Catalogue of quasars and active nuclei: 8th edn. (Garching: European Southern Observatory, ESO), ESO Sci. Rep. Ser. 18

Walker, G., Matthews, J., Kuschnig, R., et al. 2003, PASP, 115, 1023

Watson, C. L. 2006, Soc. Astron. Sci. Ann. Symp., 25, 47

Watson, C., Henden, A. A., \& Price, A. 2012, VizieR Online Data Catalog, 1, 2027

Winkler, C., Courvoisier, T. J. L., Di Cocco, G., et al. 2003, A\&A, 411, L1

Woźniak, P. R., Vestrand, W. T., Akerlof, C. W., et al. 2004, AJ, 127, 2436

Zacharias, N., Monet, D. G., Levine, S. E., et al. 2004, in BAAS, 36, AAS Meeting Abstracts, 1418

Zacharias, N., Finch, C., Girard, T., et al. 2010, AJ, 139, 2184

Zwintz, K., Weiss, W. W., Kuschnig, R., et al. 2000, A\&AS, 145, 481 